\title{
Possible $\mathrm{CO}$ clouds interacting with the SNRs G21.8-0.6 and G32.8-0.1
}

\author{
Jianjun Zhou ${ }^{1}$, Jarken Esimbek ${ }^{1}$, Xizheng Zhang $^{2}$ and \\ Hongbo Zhang ${ }^{2}$
}

${ }^{1}$ National Astronomical Observatories/Urumqi Observatory, CAS, China, 40-5 South Beijing Road, Urumqi Xinjiang 830011, China; ${ }^{2}$ National Astronomical Observatories, CAS, China, 20A, Datun Road, Chaoyang Dis, Beijing 100012.

\begin{abstract}
We have obtained the first CO(1-0) maps towards the SNRs G21.8-0.6 and G32.8-0.1, which are associated with $\mathrm{OH} 1720 \mathrm{MHz}$ masers. Based on the morphological correspondence between the radio remnant and the $\mathrm{CO}$ cloud, and the velocity and position agreement between the $\mathrm{OH}$ maser and $\mathrm{CO}$ clouds, we tentatively suggest the clouds may be interacting with G21.80.6 and G32.8-0.1. However, the shock-excited line broadening was not obvious for these two SNRs. We discuss the possible reasons for this.
\end{abstract}

Keywords. ISM:clouds - ISM: individual (G21.8-0.6, G32.8-0.1) — ISM:molecules — masers - supernova remnants

$\mathrm{OH} 1720 \mathrm{MHz}$ masers are a known signature of SNR-molecular cloud interactions. The excitation of $\mathrm{OH} 1720 \mathrm{MHz}$ maser emission requires very dense gas $\left(\sim 10^{5} \mathrm{~cm}^{-3}\right)$ with a temperature of 50-125K. A C-type shock caused by expanding supernova remnant will create such conditions (Elitzur 1976; Lockett et al. 1999). This has been confirmed by many molecular line observations (Koralesky et al. 1998; Reach \& Rho 1998; Frail \& Mitchell 1998; Reynoso et al. 2000). We took CO(1-0) observations towards the SNRs G21.8-0.6 and G32.8-0.1, both of which are associated with $1720 \mathrm{MHz} \mathrm{OH}$ masers. The sensitivity of the observation is $0.26 \mathrm{~K}$ ( $\mathrm{rms}$ ) for an integration time of 60 seconds. The results indicate that ${ }^{13} \mathrm{CO}(1-0)$ emission of these two SNRs is very weak, while no $\mathrm{C}^{18} \mathrm{O}(1$ 0 ) was detected. The CO clouds around these two SNRs are displayed in Figure 1 and Figure 2.

Based on the morphological signature of $\mathrm{CO}$ clouds, and position and velocity agreement between the $\mathrm{OH}$ maser and $\mathrm{CO}$ clouds, we tentatively suggest that cloud $\mathrm{C}$ of G21.8-0.6 and cloud F of G32.8-0.1 may be physically related to the SNR. However, we did not detect line broadening in cloud $\mathrm{F}$, and line broadening is not very strong in cloud C. A possible reason for this is that the shocked molecular gas may be highly excited, so the emission from the low-lying $\mathrm{CO}(1-0)$ transition is weak (Wilner et al. 1998). Moreover, the background cloud emission could mask such weak features. Taking into consideration the complex CO spectra, we think that confusion due to different velocity components along the line of sight plays an important role here.

\section{Acknowledgements}

This work was funded by the National Natural Science Foundation of Chinese through Grant 10203003 and the Program of the Light in China's Western Region LCRW under No.RCPY200605 and ZZBS200601. 

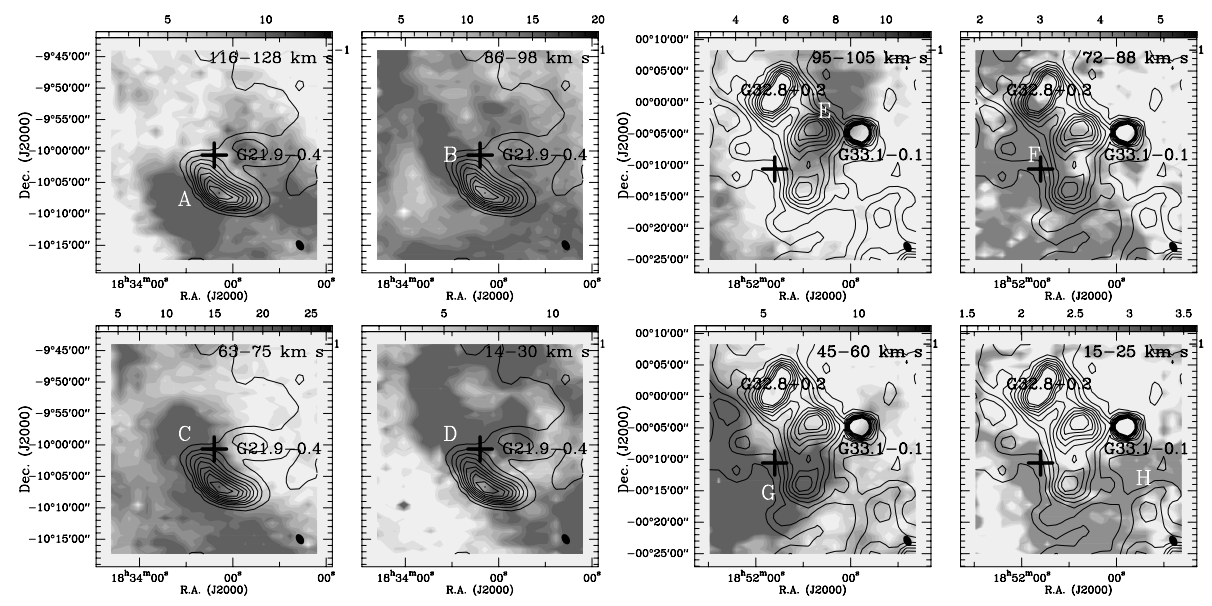

Figure 1. Left: Set of four channel maps of ${ }^{12} \mathrm{CO}(1-0)$ emission integrated over velocity ranges $116-128,86-98,63-75$ and $14-30 \mathrm{~km} \mathrm{~s}^{-1}$ toward G21.8-0.6. The grey scale is in $\mathrm{K} \mathrm{km} \mathrm{s}^{-1}$ and is shown on top of the image. The $2 \sigma$ noise level of these maps are $1.25,1.65,2.18$, and $1.29 \mathrm{~K} \mathrm{~km} \mathrm{~s}^{-1}$ respectively. The beam, $106^{\prime \prime} \times 70^{\prime \prime}$, is indicated by black filled ellipse in the bottom right corner. The contour map was obtained from the radio continuum survey of the Galactic plane at $11 \mathrm{~cm}$ wavelength(Reich et al. 1984). G21.9-0.4 is a HII region. The plus sign shows the position of $\mathrm{OH} 1720 \mathrm{MHz}$ maser.

Right: Set of four channel maps of ${ }^{12} \mathrm{CO}(1-0)$ emission integrated over the velocity ranges 95-105, 72-88, 45-60 and 15-25 $\mathrm{km} \mathrm{s}^{-1}$ toward G32.8-0.1. The grey scale is in $\mathrm{K} \mathrm{km} \mathrm{s}^{-1}$ and is shown on top of the images. The $2 \sigma$ noise level of these maps are $1.46,1.11,1.53$ and 0.99 $\mathrm{K} \mathrm{km} \mathrm{s}^{-1}$ respectively. The beam, $106^{\prime \prime} \times 70^{\prime \prime}$, is indicated by black filled ellipse in the bottom right corner. the continuum contour of G32.8-0.1 was also obtained from the radio continuum survey of the Galactic plane at $11 \mathrm{~cm}$ wavelength (Reich et al. 1984), which including two HII regions, G32.8+0.2 and G33.1-0.1. The plus sign indicates the position of $\mathrm{OH}$ maser.

\section{References}

Elitzur M., 1976, ApJ, 203, 124

Frail D. A., \& Mitchell G. F., 1998, ApJ, 508, 690

Koralesky B., Frail D. A., Goss W. M., Claussen M. J., \& Green A. J., 1998, AJ, 116, 1323

Lockett T. L., Gauthier E., \& Elitzur M., 1999, ApJ, 511, 235

Reach W. T., \& Rho J., 1998, ApJ, 507, L93

Reich W., Fürst E., Steffen P., Reif K., \& Haslam C. G., 1984, A\&AS, 58, 197

Reynoso E. M., \& Mangum J. G., 2000, ApJ, 545, 874

Wilner D. J., Reynoso S. P., \& Moffett D. A., 1998, ApJ, 115, 247 